\title{
STUDY ON OXIDATIVE STRESS BIOMARKERS IN WORKERS OF THE COLLIERY INDUSTRY
}

\author{
B. Grigorov ${ }^{1}$, M. Platikanova ${ }^{2 *}$, G. Nikolova ${ }^{1}$, Y. Karamalakova ${ }^{1}$, A. Zheleva ${ }^{1}$, V. Gadjeva ${ }^{1}$ \\ ${ }^{1}$ Department of Chemistry and Biochemistry, Medical Faculty, Trakia University, Stara Zagora, Bulgaria \\ ${ }^{2}$ Professional Diseases Section, Department of Hygiene, Infectious Diseases and Epidemiology, \\ Medical Faculty, Trakia University, Stara Zagora, Bulgaria
}

\begin{abstract}
The aim of the present research was to evaluate the effect of the surroundings on the oxidative status of workers in the mining industry. By means of two different analytical techniques have been investigated selected biomarkers of oxidative stress. By spectrophotometric methods have been studied the levels of lipid peroxidation products (MDA), catalase (CAT) activity, superoxide dismutase (SOD) activity in blood samples isolated from 30 vulcanizers exposed to trichlorethylene and 30 welders exposed to iron and manganese aerosols. Both real time biomarkers of oxidative stress such as levels of ascorbate radicals and reactive oxygen species (ROS) were also evaluated by electron paramagnetic resonance (EPR) spectroscopy. The control group consisted of 20 healthy volunteers at age close to that of the vulcanizers and welders was also studied.

By the present research were demonstrated increased levels of the studied oxidative stress biomarkers in workers from colliery "Troyanovo-1" and "Troianovo-north" to "Collieries Maritsa Iztok" EAD compared with healthy volunteers. It was supposed that increased production of reactive oxygen species might due to harmful surroundings under which the workers operate.
\end{abstract}

Key words: Trichloroethylene, vulcanisation, biomarkers, oxidative stress

\section{INTRODUCTION}

One of the main occupations in the colliery industry are machine operator curing of rubber products and welder. In the performance of their duties welders are exposed to the toxic effects of iron and manganese aerosols and vulcanizer to the toxic effects of trichloroethylene (TCE).

By inhalation of TCE is absorbed approximately $37-64 \%$ trichloroethylene by the lungs $(1,2)$ TCE is well soluble in lipids and therefore accumulates in organs containing high levels of adipose tissue, such as, the lungs, liver, kidneys and central nervous system (3). The metabolism of TCE occurs largely in the liver followed by excretion primarily in urine or unchanged in expired air (4). Based on the epidemiologic and experimental data, it is considered that TCE is potential human health hazard for noncancer

*Correspondence to: Magdalena Platikanova, Faculty of Medicine, Trakia University, Stara Zagora, Professional Diseases Section, Department of

Hygiene, Infectious Diseases and Epidemiology, mplatikanova@abv.bg toxicity to the central nervous system, kidney, liver, immune system, male reproductive system, and the developing fetus (5).

Different epidemiological studies indicate neurobehavioural, reproductive, and respiratory effects in workers exposed to manganese (6). Neurobehavioural effects most often expressed as the disturbances in the control of movements of the hand. Various studies have shown neurochemistry of manganese toxicity affected the levels of dopamine as an initial increase in dopamine followed by a longer-term decrease in humans, monkeys and rodents by exposure to manganese (7). Some authors have suggested that manganese neurotoxicity due to excessive manganese in the oxidation of dopamine, resulting in free radicals and cytotoxicity (8).

Purpose of the present study was to evaluate the oxidative status of workers in the colliery industry working under hazardous working conditions by assessing of biomarkers as lipid peroxidation products, catalase (CAT) activity, 
GRIGOROV B., et al.

superoxide dismutase (SOD) activity. The levels of "real time" biomarkers of oxidative stress such as ascorbate radicals (Asc) and reactive oxygen species (ROS) products were also studied by electron paramagnetic resonance (EPR) spectroscopy.

\section{MATERIALS AND METHODS PATIENTS AND BIOLOGICAL MATERIAL} Studies were conducted in blood samples obtained from 60 workers, 30 vulcanizers exposed to trichlorethylene and 30 welders exposed to iron and manganese aerosols from colliery "Troyanovo-1" and "Troianovo-north" to "Collieries Maritsa Iztok" EAD. The workers were divided in three groups. The $1^{\text {st }}$ consisted of 15 vulcanizers without changes in sera levels of aspartate aminotransferase (AST) and alanine aminotransferase (ALT), while the $2^{\text {nd }}$ group was vulcanizers with deviations from the reference values of serum activity of AST and ALT. As it is known AST and ALT are markers of liver function and are part of the annual prophylactic medical examinations which are carried out to workers in colliery "Troyanovo-1" and "Troianovo-north" to " Collieries Maritsa Iztok" EAD. The $3^{\text {rd }}$ group was composed of 30 welders. The control group consisted of 20 healthy volunteers at age close to that of the rest three groups.

Informed consent was obtained from all participants in the study according to the ethical guidelines of the Helsinki Declaration.

\section{LABORATORY ANALYSIS \\ Determination of the lipid peroxidation products}

The levels of MDA reactive products in the blood samples were determined by the tiobarbituric acid (TBA) method according to Plaser and Cushman 1966, with some modification by Gadjeva et al., (9). Results were expressed as micromols per mg protein MDA $(\mu \mathrm{M} / \mathrm{mgPr})$.

\section{Determination of catalase activity}

CAT activity in the erythrocyte lysates was assessed by the method described by Beers and Sizer (10). Briefly, hydrogen peroxide $(30 \mathrm{mM})$ was used as a substrate and the decrease in its concentration at $22^{\circ} \mathrm{C}$ in phosphate buffer (50mM, pH7.0) was followed spectrophotometrically at $240 \mathrm{~nm}$. One unit of CAT activity is defined as enzyme amount that degraded $1 \mu \mathrm{M} \mathrm{H}_{2} \mathrm{O}_{2}$ per minute. Results were expressed as international units per $\mathrm{mg}$ of total protein (IU/mgPr).

\section{Determination of Superoxide dismutase activity}

SOD activity was measured in the erythrocyte lysates as described by Sun et al. with minor modifications (11). The xanthine/xanthine oxidase system was used to produce the superoxide anion. This anion reduces nitroblue tetrazolium (NBT) to formazan, which is detected at $560 \mathrm{~nm}$. SOD of the sample removes the superoxide anion and inhibits the reduction. The level of this reduction is used as a measure of SOD activity (12). The concentrations of xanthine, xanthine oxidase, and nitroblue tetrazolium in the assay were respectively $50 \mu \mathrm{M}$, $10 \mathrm{U} / \mathrm{ml}$, and $0.125 \mathrm{mM}$. One unit of enzymatic activity is defined as an amount of that causes $50 \%$ inhibition of NBT reduction to formazin. The results were expressed as international units per $\mathrm{mg}$ of total protein (IU/mgPr).

\section{Electron paramagnetic resonance (EPR) studies}

All EPR measurements were performed at room temperature $\left(18-23^{\circ} \mathrm{C}\right)$ and relative humidity 40 $\%$ on a X-band EMX ${ }^{\text {micro }}$, spectrometer Bruker, Germany, equipped with standard Resonator. Experiments were carried out in triplicate. Spectral processing was performed using Bruker WIN-EPR and Simphonia software.

\section{EPR ex vivo evaluation the levels of ascorbate radicals and ROS products in the studied blood sera}

At in vivo conditions endogenic ascorbic acid can be oxidized by ROS to a stable ascorbate radical (13) and the last can be detected only by direct EPR spectroscopy which is the only method does not interfere with the biochemical processes (14). The levels of ascorbate radicals were studied according to Bailey, 2004 with some modification. Briefly, to $100 \mu \mathrm{L}$ serum was added $900 \mu \mathrm{L}$ DMSO, and after centrifugation at 4000 $\mathrm{rpm}$ for $10 \mathrm{~min}$ at $4^{\circ} \mathrm{C}$ the supernatant was collected and immediately transferred into a quartz tubes and placed in EPR cavity (15). The level of ascorbate radicals was calculated as the height of the first peak of EPR spectrum and result was expressed in arbitrary units. EPR settings were as follows: $3505.00 \mathrm{G}$ center field, $20.00 \mathrm{~mW}$ microwave power, $1.00 \mathrm{G}$ modulation amplitude, $15 \mathrm{G}$ sweep width, a receiver gain $1 \times 10^{5}, 40.96 \mathrm{~ms}$ time constant, 60.42s sweep time, 10 scans per sample. 
EPR ex vivo evaluation the levels of ROS products in the studied blood sera

To investigate in real time formation of reactive oxygen species (ROS) in the sera of the workers and controls was used EPR spectroscopy combined with ex vivo PBN spin trapping. The spin trap PBN, upon reaction with unstable radicals forms a relatively stable spin adduct that can be subsequently detected by EPR spectroscopy. The levels of ROS were determined according Shi et al., 2005 with modifications by Zheleva et al., 2011 (16). Briefly, to $100 \mu \mathrm{L}$ serum was added $900 \mu \mathrm{L} 50$ $\mathrm{mM}$ PBN dissolved in DMSO and after centrifugation at $4000 \mathrm{rpm}$ for $10 \mathrm{~min}$ at $4^{\circ} \mathrm{C} \mathrm{EPR}$ spectrum of the supernatant was recorded. The levels of ROS products were calculated as double integrated plots of EPR spectra and results were expressed in arbitrary units. EPR setting were as follows: Center field - 3503.73 G, Microwave power - $20.00 \mathrm{~mW}$, Modulation amplitude - 5G, Sweep width $-50 \mathrm{G}$, Receiver gain $-1 \times 10^{5}$, Time constant $-81.92 \mathrm{~ms}$, Sweep time -125.95 sec. Scans per sample -5 scans.

\section{Statistical analysis}

Statistical analysis was performed with Statistica 7, StaSoft, Inc. And the results were expressed as means \pm S.E. Statistical analysis was performed with Student's t-test. $\mathrm{P}<0.05$ was considered statistically significant.

\section{RESULTS}

On Figure 1 are shown MDA levels measured in the blood samples of the workers and controls enroled in this study. As can be seen, in the $1^{\text {st }}$ group, MDA levels were significantly higher than those of the control group $(4.104 \pm 0.121 \mu \mathrm{M}$ vs. $3.56 \pm 0.07 \mu \mathrm{M}, \mathrm{P}<0.005)$. The levels of MDA in the $2^{\text {nd }}$ group were also significantly higher comparing to those of control group (4.83 \pm $0.19 \mu \mathrm{M}$ vs. $3.56 \pm 0.07 \mu \mathrm{M}, \mathrm{P}<0.0002)$. Only in the $3^{\text {rd }}$ group, the levels of MDA were significantly decreased compared to those of the control group $(3.15 \pm 0.05 \mu \mathrm{M}$ vs. $3.56 \pm$ $0.07 \mu \mathrm{M}, \mathrm{P}<0.002)$.

On Figure $\mathbf{2}$ are presented the levels of erythrocyte CAT activity measured in the four groups. As is seen, statistical significantly higher levels of CAT activity were found for the $1^{\text {st }}$ and $2^{\text {nd }}$ group comparing to those of the control group $(21839.23 \pm 2512.064 \mathrm{IU} / \mathrm{gHb}$ vs. $4518.03 \pm$ $658.455 \mathrm{IU} / \mathrm{gHb}, \mathrm{P}<0.0001)$ and $(29159.33 \pm$ 2931.695 vs. $4518.03 \pm 658.455 \mathrm{IU} / \mathrm{gHb}, \mathrm{P}<$ $0.00005)$, respectively. In the $3^{\text {rd }}$ group, the erythrocyte CAT activity was significantly decreased in comparison with the control group $(1772.79 \pm 173.539 \mathrm{IU} / \mathrm{gHb}$ vs. $4518.03 \pm$ $658.455 \mathrm{IU} / \mathrm{gHb}, \mathrm{P}<0.01)$.

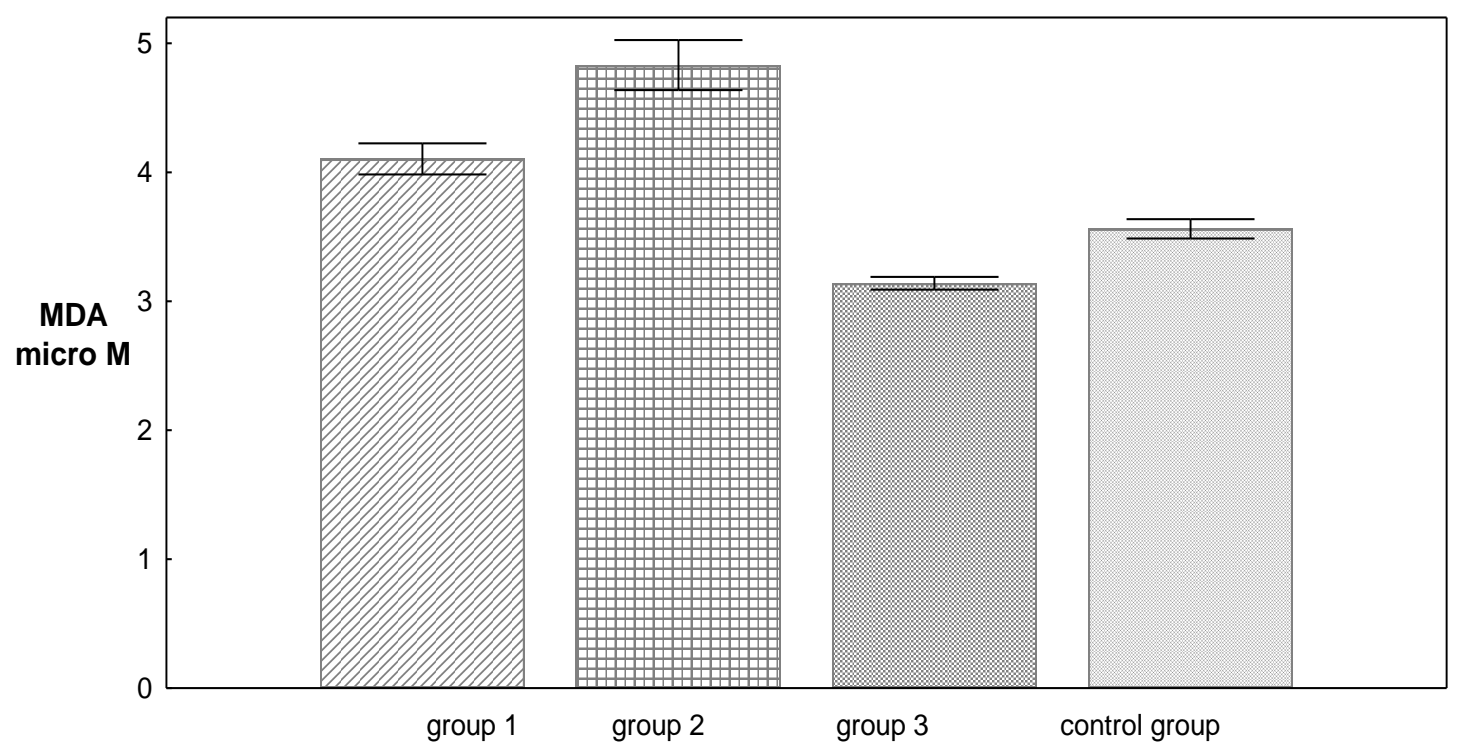

Figure 1. Levels of MDA reactive products in blood samples of the workers and healthy controls. Results are expressed as $\mu \mathrm{M}$ MDA products per mg protein. 


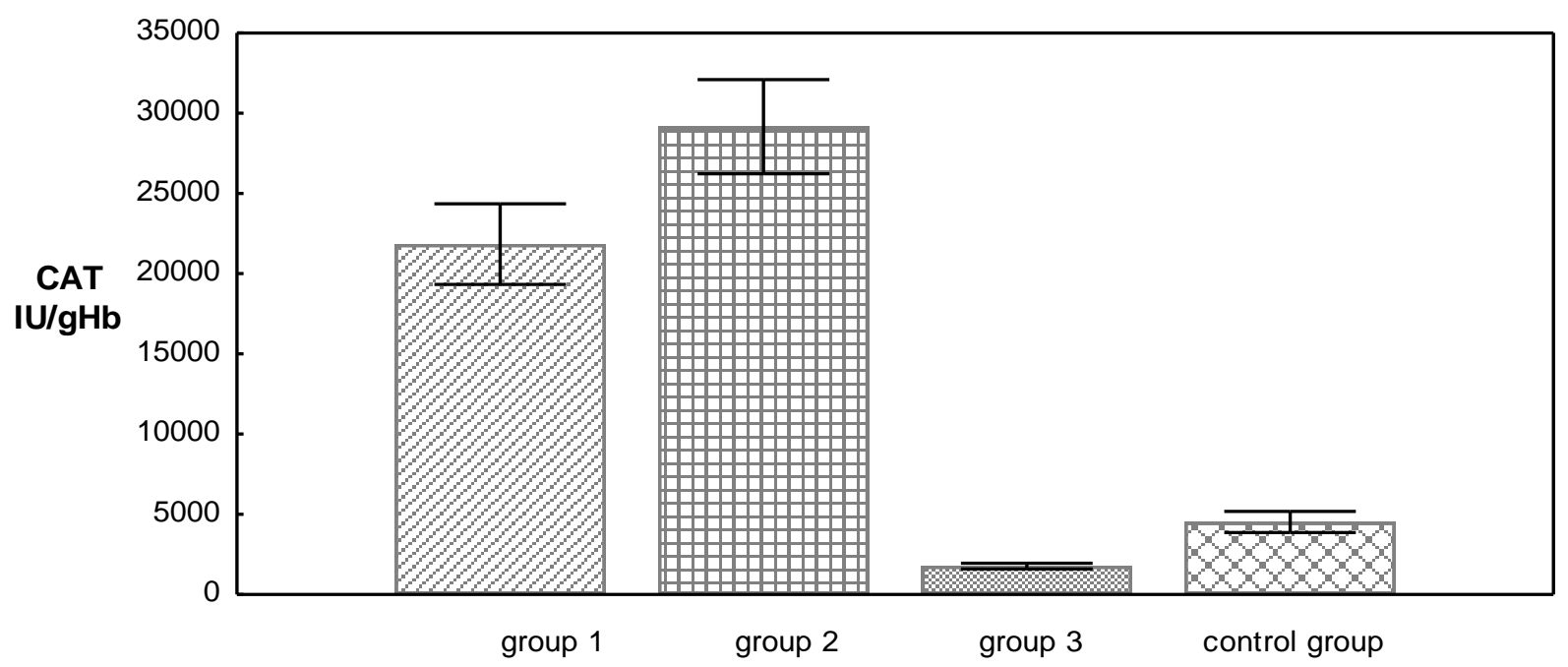

Figure 2. Levels of CAT activity in the erythrocyte lysates expressed as IU/gHb.

The erythrocyte SOD activity in all groups of workers was significantly higher compared with the control group. The levels of erythrocyte SOD activity in the $1^{\text {st }}$ and $2^{\text {nd }}$ group were significantly higher, comparing with the controls, respectively $(369.408 \pm 58.563 \mathrm{IU} / \mathrm{gHb}$ vs. $111.263 \pm 10.899 \mathrm{IU} / \mathrm{gHb}, \mathrm{P}<0.01)$ and
$(677.527 \pm 65.740 \mathrm{IU} / \mathrm{gHb}$ vs. $111.263 \pm 10.899$ $\mathrm{IU} / \mathrm{gHb}, \mathrm{P}<0.00001)$. There was a significant increase in erythrocyte SOD activity of the $3^{\text {rd }}$ group comparing to that of the control group $(290.750 \pm 28.353 \mathrm{IU} / \mathrm{gHb}$ vs. $111.263 \pm 10.899$ $\mathrm{IU} / \mathrm{gHb}, \mathrm{P}<0.0001)$.

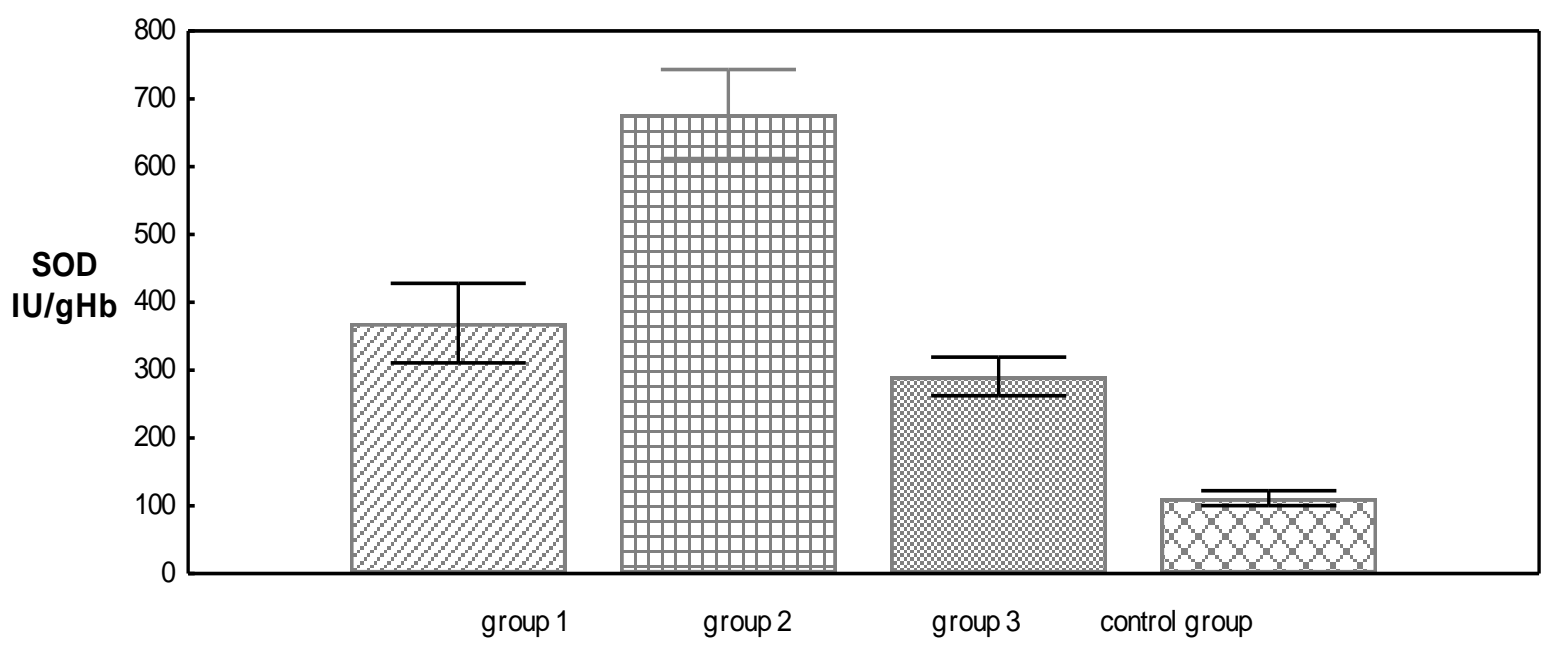

Figure 3. Levels of SOD activity in the erythrocyte lysates expressed as IU/gHb.

Results from the levels of ascorbate radicals measured in the studied sera are presented on Figure 4. In all studied samples was registered EPR doublet signal typical for the ascorbate radical (EPR spectrum is not shown). The level of ascorbate radicals was calculated from the height of first peak of the EPR doublet signal. The levels of ascorbate radicals registered in sera isolated from the workers of the $1^{\text {st }}$ and $3^{\text {rd }}$ group were significantly higher, compared to that of the healthy controls: $3386542 \pm 153131.9$ arb.units vs. $2315745 \pm 82068.8$ arb.units, $\mathrm{P}<0.0001$ and $3513047 \pm 129523.4$ arb.units vs. $2315745 \pm$ 82068.8 arb.units, $\mathrm{P}<0.00001$, respectively. There was also found a significant increase in the levels of ascorbate radicals in the $2^{\text {nd }}$ group compared to the control group (3012881 \pm 118757.8 arb.units vs. $2315745 \pm 82068.8$ arb.units, $\mathrm{P}<0.001)$. 


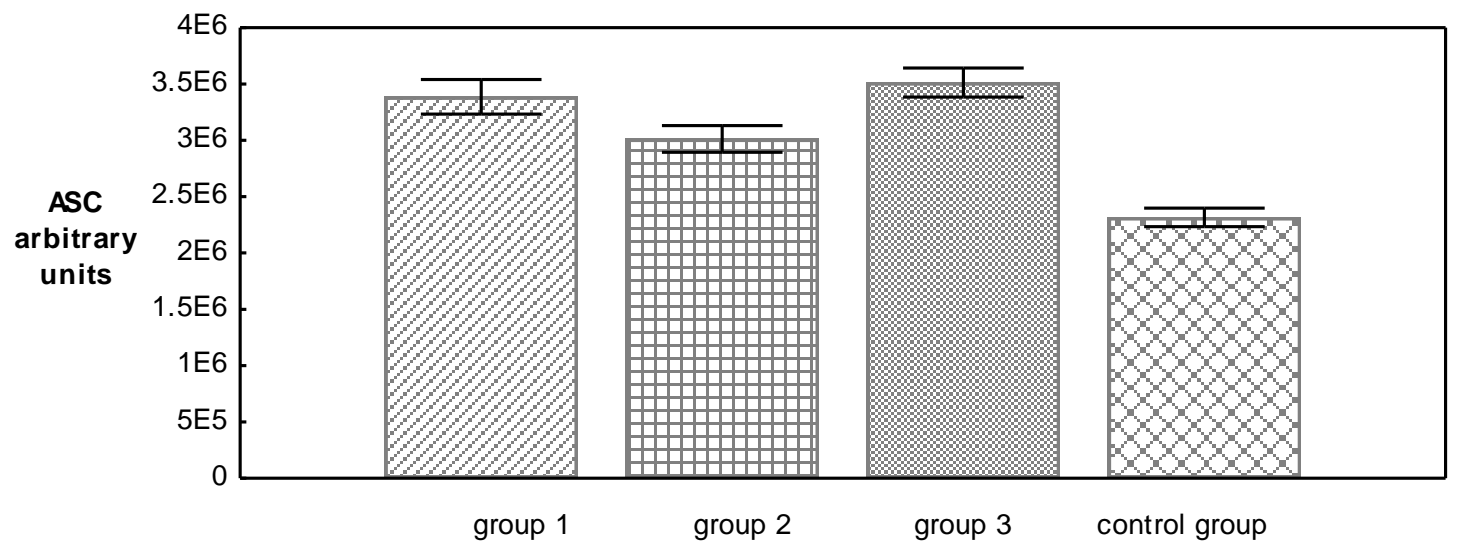

Figure 4. The levels of Ascorbate radicals (expressed in arb. units) registered in blood samples of the studied and control groups

Results from the levels of ROS products measured in the studied sera are presented on Figure 5. In all studied samples was registered EPR spectrum of the typical PBN spin adduct consisting of six spectral lines (EPR spectrum is not shown). Based on the values of the calculated hyperfine splitting constants $\left(\mathrm{a}^{\mathrm{N}}=13.88 \mathrm{G}\right.$ and $\mathrm{a}_{\beta}{ }^{\mathrm{H}}=2.35 \mathrm{G}$ ) PBN spin adduct and that the sera were mixed with DMSO solution of PBN in aerobic conditions, the generated free radical species were identified as secondary oxygen centered alkoxy radicals (17). Levels of ROS products were calculated after double integration of the plot of the corresponding EPR spectrum.
As is seen on Figure 5 the levels of ROS products registered in the blood samples isolated from the $1^{\text {st }}$ group of workers were not significantly different from that of the control group $(2.527 \pm$ 0.146 arb.units vs. $2.432 \pm 0.112$ arb.units, $\mathrm{P}>$ $0.05)$. There was a significant decrease in the levels of ROS products calculated for the $2^{\text {nd }}$ group compared to the control group $(1.910 \pm$ 0.179 arb.units vs. $2.432 \pm 0.112$ arb.units, $\mathrm{P}<$ 0.05). Contrary, the levels of ROS products registered in the $3^{\text {rd }}$ group were significantly higher than those of the control group $(3.100 \pm$ 0.244 arb.units vs. $2.432 \pm 0.112$ arb.units, $\mathrm{P}<$ $0.05)$.

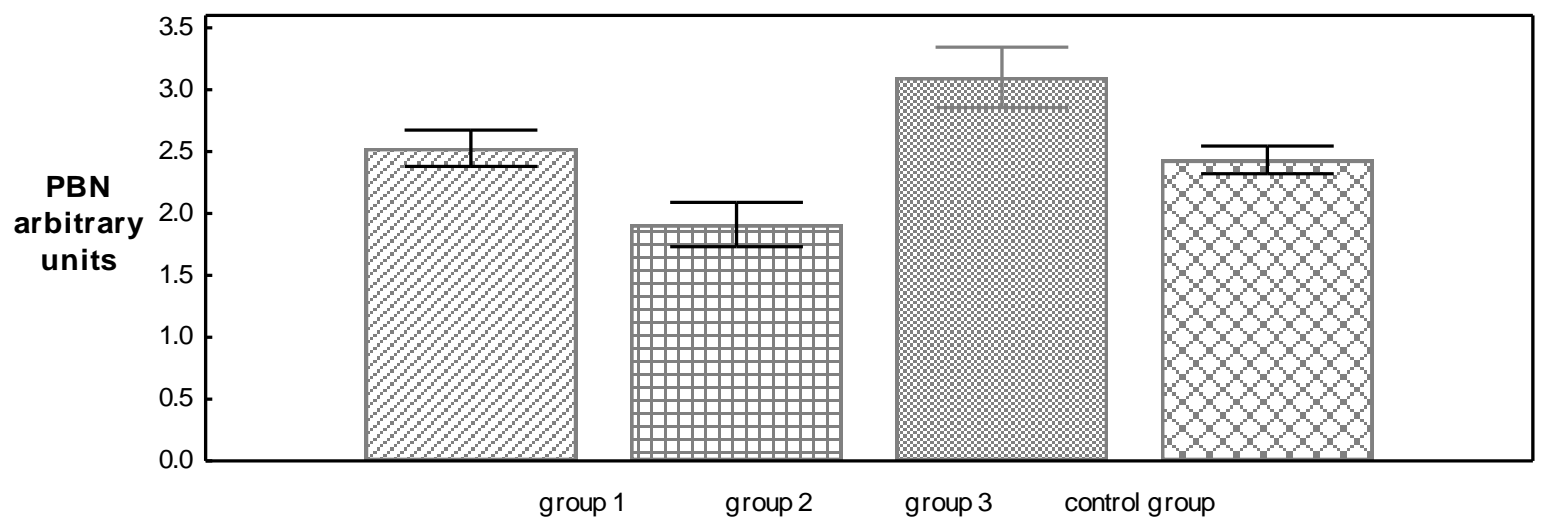

Figure 5. The levels of ROS products (expressed in arb. units) registered in blood samples of the studied and control group

\section{DISCUSSION}

The toxicity of TCE is due to its reactive metabolites derived from the reaction of glutathione conjugating with TCE (18). there are a number of reports in support of this statement. Other authors have reported that TCE-induced oxidative stress in the livers of mice is close related to the metabolism of TCE to trichloroacetic acid $(19,20)$. An imbalance between the production of reactive oxygen species and cellular antioxidants has been demonstrated by another study conducted on mice treated with TCE (21)There are a number of epidemiology studies in workers showing the relationship between TCE exposure and renal cell cancer mortality and incidence, but has not been established the mechanism for induction of malignant disease (22). Our results showed a significant increase in the levels of MDA, CAT, SOD and ascorbate radicals in workers from 
group 1 and 2 compared with the control group. It is not surprise that the levels of MDA, SOD and CAT activity determined for the $2^{\text {nd }}$ group were higher than those determined for the $1^{\text {st }}$ group. Workers enrolled in $2^{\text {nd }}$ group have deviations from the reference values of the serum enzyme activity of AST and ALT. This finding means that the level of the oxidative processes in the livers of workers in the $2^{\text {nd }}$ group is higher comparing to that of the $1^{\text {st }}$ group consisted of workers without changes in sera levels of AST and ALT. As it is known AST and ALT are markers of liver function. It should be mentioned, that with an exception for the ROS levels measured in the $2^{\text {nd }}$ group, the levels of the ascorbate radicals and ROS products in the three groups of workers were higher than those of the healthy controls. This result confirms that oxidative processes are in progress at the time of the study. There exist sufficiently reports which indicate that long-term exposure to TCE may lead to hepatotoxicity, nephrotoxicity and malignancies $(23,24,25)$ It is also known that free radical damage have a role in the initiation of carcinogenesis. However, there is no evidence about the relation between reactive oxygen species caused by TCE and initiation of carcinogenesis. Moreover, it is not known whether oxidative stress is a direct consequence of the metabolism of or resulting from TCEinduced hepatotoxicity and nephrotoxicity.

The results of the $3^{\text {rd }}$ group consists of welders exposed to toxic gases showed that the levels of SOD, ascorbate radicals and ROS products were elevated comparing to the controls. It is obvious that a permanent oxidative process, in the workers of the $3^{\text {rd }}$ group is available. On the other hand statistical significant decrease in the levels of MDA measured for the same group means that oxidative damages are overcame by the antioxitant defense of the workers in that group. Insufficient data are available for the oxidative status in workers exposed to manganese. However, there are several epidemiological studies of workers have provided consistent evidence indicating that neurotoxicity is associated with low-level manganese exposure, but it is not exactly elucidated mechanism of the toxicity (7).

\section{CONCLUSION}

By the present paper have been demonstrated increased levels of the studied oxidative stress biomarkers in blood samples of workers from colliery "Troyanovo-1" and
"Troianovo-north" to "Collieries Maritsa Iztok" EAD, in comparison with those of the healthy volunteers. It was supposed that harmful surroundings under which the workers operate might be the reason for the increased production of reactive oxygen species.

\section{ACKNOWLEDGEMENTS}

This article is the result of research project № 3 of 2011 of the Faculty of Medicine, Trakia University, Stara Zagora „Research in Oxidative Stress Biomarkers in People Working with Adhesives Containing Trichlorethylene in the Ore/mining Industry. Identification of Dependencies between the Biomarker Levels and Some Biochemical Indicators", headed by Prof. Dr. in Chemical Sciences V. Gadzheva.

\section{REFERENCES}

1. Agency for Toxic Substances and Disease Registry (ATSDR) Toxicological Profile for Trichloroethylene. US department of Health and Human Services. Atlanta, US, 1997.

2. International Programme on Chemical Safety (IPCS) Trichloroethylene. Environmental Health Criteria 50. WHO. Geneva, 1985.

3. Dallas, C. E., J. M. Gallo, et al. "Physiological pharmacokinetic modeling of inhaled trichloroethylene in rats." Toxicology \& Applied Pharmacology 110(2): 303-14, 1991.

4. Yu, D., Bove, F., etc., Agency for Toxic Substances and Disease Registry, Trichloroethylene Toxicity, Case Studies in Environmental Medicine (CSEM), , November 8, 2010

5. Chiu, A. W., Barone, S., etc.,toxicological review of trichloroethylene, (cas no. 79-01-6), U.S. Environmental Protection Agency Washington, DC, September 2011.

6. Mergler, D. ET AL. Nervous system dysfunction among workers with long-term exposure to manganese. Environmental research, 64: 151-180,1994.

7. World Health Organization Regional Office for Europe Copenhagen, Air Quality Guidelines for Europe Second Edition, Chapter 6.8, ISSN 0378-2255, 2001.

8. Donaldson, J. ET AL. Manganese neurotoxicity: a model for free radical mediated neurodegeneration Canadian journal of physiology and pharmacology, 60: 1398-1405, 1982.

9. Gadjeva, V., Kuchukova, D., Georgieva, RInfluence of polychemotherapy on the antioxidant levels and lipid peroxidation in 
patients with lymphoproliferative diseases. Comparative Clinical Pathology 14, (1)1318, 2005.

10.Beers, R., Sizer, T., Spectrophotometric method for measuring the breakdown of hydrogen peroxide by catalase. $J$ Biol Chem, 195:133-138, 1952.

11.Sun, Y., Oberley, LW., Li, Y. A simple method for clinical assay of superoxide dismutase. Clin Chem 34:497-500, 1988.

12.Gadjeva, V., Dimov, A., Georgieva, N., Influence of therapy on the antioxidant status in patients with melanoma. J Clin Pharm Ther. (2008), 33(2)

13. Armstrong, D. Methods in Molecular Biology, vol. 108, Free radical and antioxidant protocols, Humana Press Ins., Totowa, NJ, USA, 1999.

14.Halliwell, B. and Whiteman, M. Measuring reactive species and oxidative damage in vivo and in cell culture: how should you do it and what do the results mean? Brit.J.Pharmacol., 142(2):231-255,

DOI: 10.1038/sj.bjp.0705776, 2004.

15.Bailey D. Ascorbate, blood-brain barrier function and acute mountain sickness: a radical hypothesis. Wilderness Environ Med.,15: 231-233, 2004

16.Zheleva, A., A. Tolekova, Y. Karamalakova, G. Nikolova, V. Gadjeva. Ex vivo Evaluation of oxidative stress caused by 2chlorethylynitrosourea antitumor agent in mice liver tissue- a comperative study by two different methods. III Internatinal conference of oxidative stress. Havana-Redox., Cuba, 2011-27-29.01, Revista Cubana de Farmacia, Vol.45 supl.1, AB., p-1-139, 2011.

17.Luo Yi, Su Y, Lin R, Shi H, Wang X, 2Chlorophenol induced ROS generation in fish Carassius auratus based on the EPR method. Chemosphere, 65 (6): 1064-1073, 2006.

18.Geoptar AR, Commandeur JNM, Van Ommen B, Van Bladeren PJ, Vermenten
NPE: Metabolism and kinetics of trichloroethylene in relation to toxicity and carcinogenicity. Relevance of the mercapturic acid and pathway. Chem Res Tocicol , 8(1):321, 1995.

19.Laughter, A. R., Dunn, C. S., Swanson, C. L., Howroyd, P., Cattley, R. C., and Corton, J. C. Role of the peroxisome proliferator-activated receptor alpha (PPARalpha) in responses to trichloroethylene and metabolites, trichloroacetate and dichloroacetate in mouse liver. Toxicology 203, 83-98, 2004.

20.Nakajima, T., Kamijo, Y., Usuda, N., Liang, Y., Fukushima, Y., Kametani, K., Gonzalez, F. J., and Aoyama, T. Sex-dependent regulation of hepatic peroxisome proliferation in mice by trichloroethylene via peroxisome proliferator-activated receptor alpha (PPARalpha). Carcinogenesis 21, 677-682, 2000.

21.Cojocel, C., Beuter, W., Muller, W., Mayer, D., Lipid peroxidation: a possible mechanism of trichloroethylene induced nepherotoxicity. Toxicol, 55(1-2):131-141, 1989.

22.Lock, E. A. and Reed, C. J., Trichloroethylene: Mechanisms of Renal Toxicity and Renal Cancer and Relevance to Risk Assessment, Toxicological sciences 91(2), 313-331, 2006.

23.Bull RJ. Mode of action of liver tumor induction by trichloroethylene and its metabolites, trichloroacetate and dichloroacetate. Environ Health Perspect 108(suppi 2):241-259, 2000.

24.Pastino, G. M., Yap, Y. W., and Carroquino, M., Human Variability and Susceptibility to Trichloroethylene, Environmental Health Perspectives * Vol 108, Supplement 2*, May 2000.

25.Lash LH. Modes of action for kidney tumorigenisis. Environ Health Perspect 108(suppi 2):225-240, 2000. 